\title{
Long-term Results of Mini Asymmetric Radial Keratotomy and Corneal Cross-linking for the Treatment of Keratoconus
}

\author{
Marco Abbondanza ${ }^{1}$, Gabriele Abbondanza ${ }^{1,2}$, Valentina De Felice ${ }^{1}$, Zoie Shui-Yee Wong ${ }^{3}$ \\ ${ }^{1}$ Abbondanza Eye Centers, Rome and Milan, Italy \\ ${ }^{2}$ Centre for International Security Studies, The University of Sydney, Sydney, Australia \\ ${ }^{3}$ Graduate School of Public Health, St. Luke's International University, Tokyo, Japan
}

Purpose: To investigate the long-term results (at least 5 years of follow-up) of the mini asymmetric radial keratotomy (MARK) and corneal cross-linking (CXL) combined intervention, also known as the 'Rome protocol,' for patients with progressive stage I and II keratoconus and contact lens intolerance.

Methods: This was a retrospective observational case series. Fifteen eyes of 12 patients were evaluated, with a mean follow-up of 6.9 years. To assess the efficacy and stability of the MARK + CXL combined protocol, best spectacle-corrected visual acuity, mean pachymetry, and mean keratometry were recorded preoperatively and at least 1,3 , and 5 years postoperatively. Statistical analysis was performed using the R platform and involved the Wilcoxon signed-rank and Kruskal-Wallis non-parametric tests.

Results: Best spectacle-corrected visual acuity improved for all patients, from $0.46 \pm 0.69$ logarithm of the minimum angle of resolution (20 / 60) to $0.15 \pm 0.69$ logarithm of the minimum angle of resolution $(20 / 30, p=$ 0.0006 ), while mean pachymetry increased in $93 \%$ of patients, from $442.80 \pm 61.02$ to $464.50 \pm 62.72 \mu \mathrm{m}$ ( $p$ $=0.003$ ). Lastly, mean keratometry improved in $87 \%$ of patients after 6.9 years of observation from $48.82 \pm$ 5.00 to $43.25 \pm 3.58$ diopters $(p=0.008$ ). No intraoperative or postoperative complications were observed.

Conclusions: The MARK + CXL combined protocol was effective in treating keratoconus by halting corneal thinning and bulging. In addition, this procedure significantly improved visual acuity based on long-term follow-up data. Analysis of data from a larger cohort of patients would be useful to support these findings.

Key Words: Corneal cross-linking, Keratoconus, Mini asymmetric radial keratotomy, Rome protocol

Keratoconus (KC) is a non-inflammatory corneal dystrophy that often occurs in the second decade of life with a bilateral onset. Clearly identified in 1854 by John Nottingham

Received: March 6, 2018 Accepted: June 28, 2018

Corresponding Author: Marco Abbondanza, MD. Abbondanza Eye Center, Via Luigi Bodio 72, Rome 00191, Italy. Tel: 39-6-320-7111, E-mail: info@abbondanza.org
[1], this corneal ectasia results in asymmetric and progressive corneal distortion and thinning, altered refractive powers, and decreased vision [2]. In $15 \%$ to $20 \%$ of cases, moreover, corneal transplantation may be required. This intervention is performed to replace all corneal layers penetrating keratoplasty or, in less advanced stages, selected corneal layers deep anterior lamellar keratoplasty [3].

Despite the undoubtable usefulness and constant refinement of corneal transplantation, however, it remains an in- 
vasive procedure that exposes the patient to varying degrees of rejection risk and, importantly, does not offer a permanent solution, as a corneal graft's mean survival is 16.88 years [4]. For these and other reasons, a number of conservative techniques has been developed or adapted during the last two decades: corneal cross-linking (CXL) [5], intrastromal corneal ring segments (ICRS) [6], mini asymmetric radial keratotomy (MARK) [7,8], circular keratotomy (CK and FemtoCK) [9], conductive keratoplasty (CKP) [10] and radial keratotomy (RK)/mini-radial keratotomy [11-13]. CXL, in particular, has become the gold standard in the treatment of progressive $\mathrm{KC}$ as it reinforces the weakening corneal structure, thus halting ectasia.

Apart from a slight degree of corneal flattening with the 'epi-off' (Dresden protocol) technique, however, it does not significantly improve a patient's visual acuity [14], a condition that has led to a number of CXL-combined procedures. Combined protocols discussed in the medical literature include CXL paired with photorefractive keratectomy (PRK) (Athens protocol) [15], femtosecond laser-assisted in situ keratomileusis (FemtoLASIK) [16], ICRS [17], phakic intraocular lenses [18], CKP [19], and MARK [20]. The latter combined intervention, the 'Rome protocol,' was the focus of this study, the aim of which was to investigate the effectiveness of this protocol for disease stabilization and improvement of visual acuity based on long-term follow-up data.

\section{Materials and Methods}

This was a retrospective, observational, case series of patients who underwent combined MARK + CXL intervention performed by the same surgeon (MA) with the same instruments in two different centers who were followed-up tor several years. Liınical selection criteria includea progressive stage I and II KC (Amsler-Krumeich classification) and contact lens intolerance. Patients with any chronic or recurrent ocular infections were excluded. All patients underwent a complete clinical examination prior to the intervention. Ethics approval was provided by the director of the Abbondanza Eye Centers (MA), and written informed consent for both procedures was required as a part of our preoperative routine. The research was performed in accordance with the principles of the Declaration of Helsinki.

For the purposes of this study, best spectacle-corrected visual acuity (BSCVA), mean pachymetry, and mean ker-

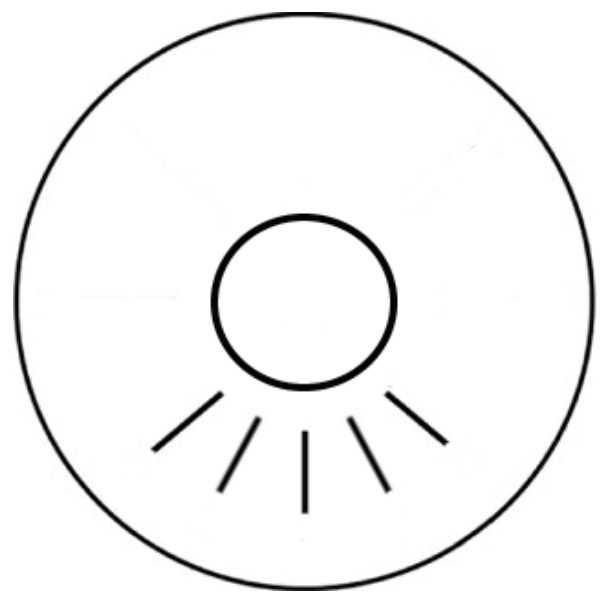

Fig. 1. Diagram of mini asymmetric radial keratotomy mini-incisions.

atometry (Kavg) were recorded with a Pentacam (Oculus Optikgerate $\mathrm{GmbH}$, Wetzlar, Germany) preoperatively and at least 1,3 , and 5 years following the last of the combined operations (CXL). Steady and/or improved pachymetric and keratometric values have been used as indicators of successful KC stabilization, while an increase in BSCVA is a standard indicator of improved visual acuity.

\section{MARK surgery}

Once the inclusion/exclusion criteria were satisfied, preparation for MARK was initiated by devising a surgical plan. Because MAPK surgery comprises a small series (3 to 5) of tomography- and topography-guided mini-incisions to circa $80 \%$ of corneal thickness, performed only on the portion of the cornea where $\mathrm{KC}$ is manifest (Fig. 1), factors that were carefully considered included the designation of the corneal clear zone, which was to be left as large as possible, along with determination of the number, position, length, and depth of the mini-incisions. MARK's conservative nomogram is based on a modified version of Lindstrom's [21] so that it performs shorter (1.75 to $2.25 \mathrm{~mm}$ instead of $3 \mathrm{~mm}$ ) and asymmetric (sectorial) mini-incisions. A diamond knife (Meyco; Anton Meyer \& Co., Biel, Switzerland) and custom-made double concentric corneal markers (Abbondanza markers) (Fig. 2), were used and incisions were limited to a maximum of 7.5 to $8 \mathrm{~mm}$ on the external end and a minimum of 3.5 to $4 \mathrm{~mm}$ on the internal end. Topical anesthesia was applied prior to the treatment (benoxinate chloride $0.4 \%$; Alfa Intes Srl, Casoria, Italy). Follow- 
ing the intervention, a bandage contact lens was placed (CooperVision, Pleasanton, CA, USA) and topical antibiotics (levofloxacin hemihydrate $0.5 \%$, Alfa Intes Srl) and non-steroidal anti-inflammatory drops (diclofenac sodic $0.1 \%$; Thea Laboratoires, Clermont-Ferrand, France) were prescribed.

\section{CXL treatment}

After repeating the same series of examinations described above, CXL was performed when complete consolidation of the mini-incisions was observed with biomicroscopy to avoid possible reopening of such mini-incisions during the treatment. Complete consolidation was observed on average about 12 months after MARK surgery. The standard Dresden Protocol for CXL was followed, and after topical anesthesia, the corneal epithelium was removed with an ophthalmic scalpel (MicroFeather; Feather Safety Razor, Osaka, Japan) and riboflavin eye drops were applied on the corneal stroma 30 minutes before the procedure 10 times at 3-minute intervals. Riboflavin was then applied six times at 5-minute intervals during UV-A irradiation (UV-X 1000; IROC AG, Zurich, Switzerland). Eyelids were kept open using ophthalmic specula. Total UV-A exposure time was 30 minutes and postoperative therapy was performed as described for MARK surgery.

\section{Statistical analysis}

All data were collected in standardized study spreawdsheets and entered into Excel 2010 (Microsoft, Redmond,

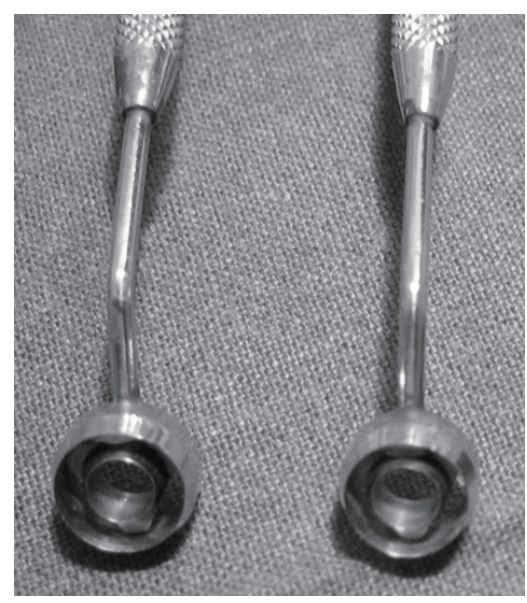

Fig. 2. The Abbondanza markers.
WA, USA). Analyses were performed using the R platform (R Development Core Team, Vienna, Austria), version 3.3.2, 64 bit, with the 'ggplot2,' 'Rmisc,' and 'reshape2' packages. Non-parametric significance tests were used, specifically the Wilcoxon signed-rank test and the Kruskal-Wallis test, and a $p$-value of $<0.05$ was considered statistically significant. Visual acuity was measured according to a decimal scale and then converted to the logarithm of the minimum angle of resolution (logMAR). Lastly, means and standard deviations were computed for all numerical data analyzed with R.

\section{Results}

Among all patients that underwent the MARK + CXL combined procedure, fifteen eyes of 12 patients were selected due to availability of a minimum of 5 years of follow-up data after the last intervention. Mean follow-up was 6.9 years. The cohort of patients comprised eight males and four females with a mean age of 34.8 years. No patients required corneal transplantation. The first parameter we employed to evaluate effective $\mathrm{KC}$ halting, namely mean keratometry, showed considerable improvements over time. Kavg improved in $87 \%$ of patients after a mean of 6.9 years of observation, from $48.82 \pm 5.00$ to $43.25 \pm 3.58$ diopters ( $p$ $=0.008)$ (Fig. 3).

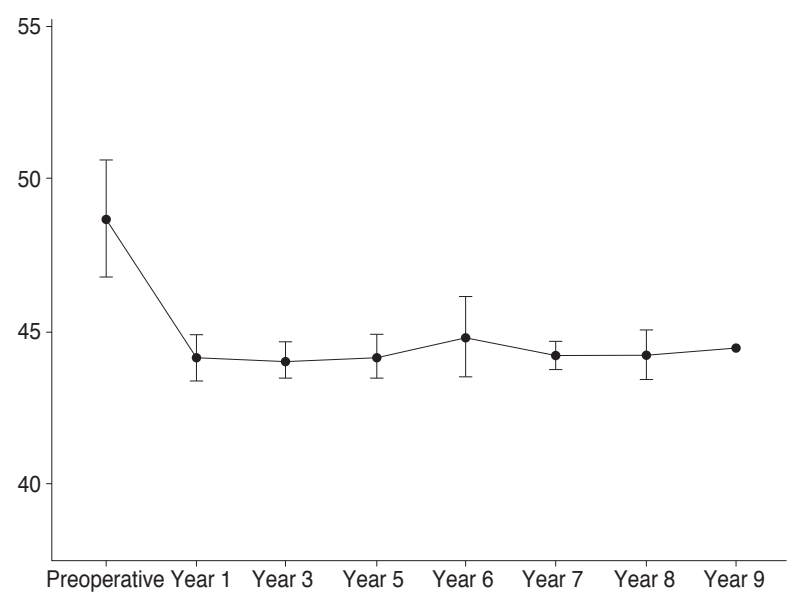

Fig. 3. Mean keratometry changes (with confidence intervals) before and after surgery. A Kruskal-Wallis non-parametric test showed that mean keratometry values do not have identical data distributions in the follow-up period $(p=0.175)$. A Wilcoxon signed-rank test indicates that postoperative Kavg values after 7 years are significantly lower than preoperative values $(p<0.05)$. 


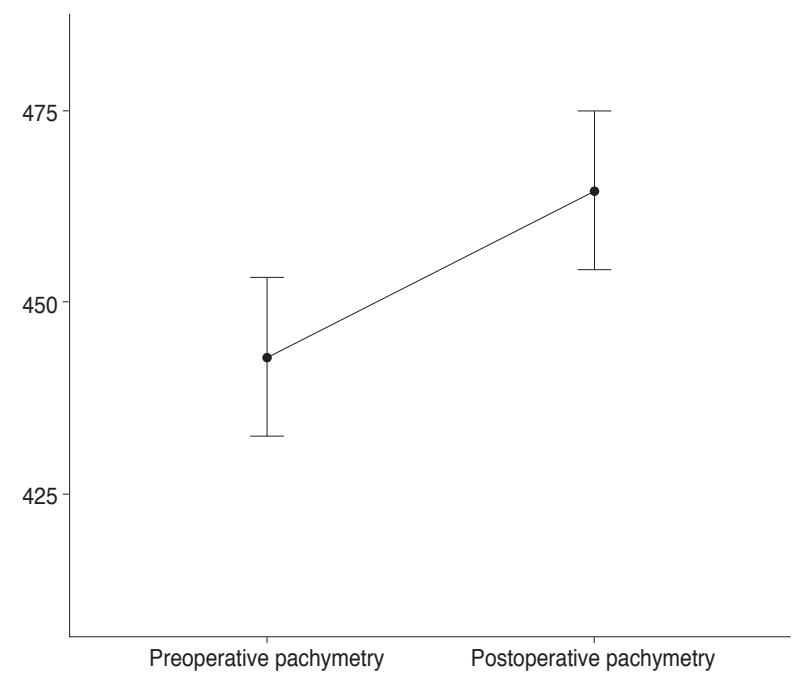

Fig. 4. Mean pachymetry (with $95 \%$ confidence intervals) before and after surgery. A Wilcoxon signed-rank test showed that postoperative pachymetry is significantly higher than preoperative pachymetry $(p<0.005)$.
A

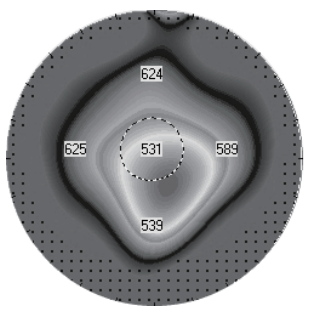

B

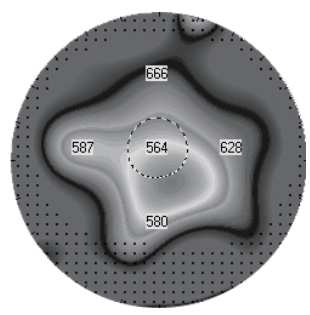

Fig. 5. Pachymetric map (A) before and (B) after surgery, showing a general improvement in corneal thickness.

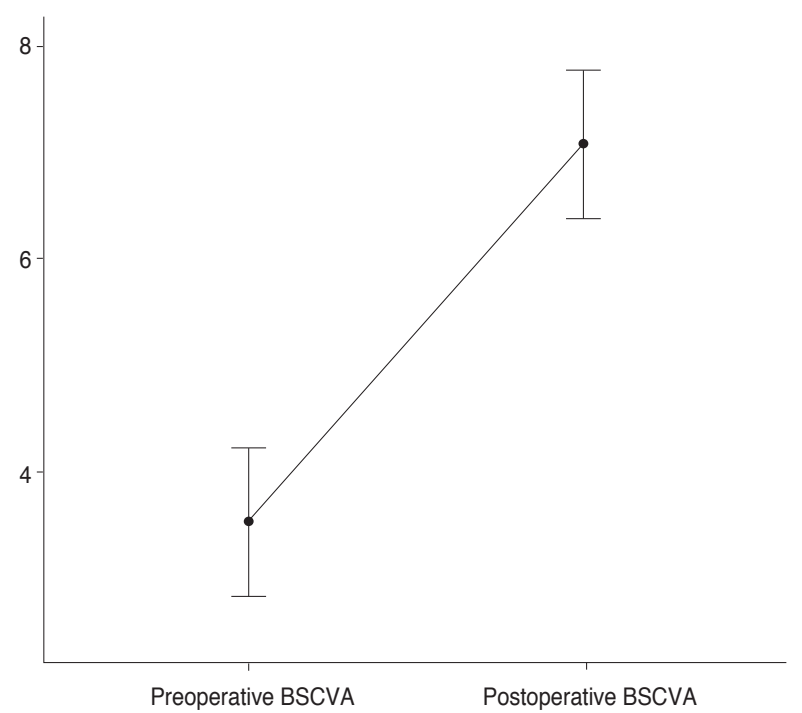

Fig. 6. Mean best spectacle-corrected visual acuity (BSCVA) (on a decimal scale with $95 \%$ confidence intervals) before and after surgery. A Wilcoxon signed-rank test shows that postoperative BSCVA is significantly higher than preoperative BSCVA $(p<0.005)$.
Table 1. Aggregate preoperative and postoperative data with statistically-significant outcomes

\begin{tabular}{lccc}
\hline Cause & Before & After & $p$-value \\
\hline Kavg $(1 \mathrm{yr})$ & 48.8 & 44.25 & 0.0007 \\
Kavg $(5 \mathrm{yr})$ & 48.8 & 44.28 & 0.004 \\
Kavg (7 yr) & 48.8 & 43.25 & 0.008 \\
Mean pachymetry & 442.8 & 464.5 & 0.003 \\
BSCVA & $0.46(20 / 60)$ & $0.15(20 / 30)$ & 0.0006 \\
\hline
\end{tabular}

Kavg = mean keratometry; BSCVA = best spectacle-corrected visual acuity.

Corneal thickness also improved noticeably; mean pachymetry increased in $93 \%$ of patients from $442.80 \pm 61.02$ to $464.50 \pm 62.72 \mu \mathrm{m}$ ( $p=0.003$ ) (Fig. 4, 5). Lastly, postoperative visual acuity as measured by BSCVA improved in all patients from $0.46 \pm 0.69 \log$ MAR $(20 / 60)$ to $0.15 \pm$ $0.69 \log$ MAR $(20 / 30, p=0.0006)$ (Fig. 6). Aggregate preoperative and postoperative data with relevant $p$-values are presented in Table 1, while key indicators for each patient are shown in Table 2. The original dataset is available upon request from the corresponding author.

\section{Discussion}

Four main conclusions can be drawn from our study. First, combined MARK and CXL appeared to be effective in treating both progressive $\mathrm{KC}$ (mean keratometry and pachymetry improved) and improving patients' visual acuity (BSCVA increased). Second, the combined protocol appeared to provide predictable outcomes, as mean keratometry, mean pachymetry, and visual acuity improved in $87 \%$, $93 \%$, and $100 \%$ of cases respectively. Third, the protocol proved to be safe - no intraoperative or postoperative complications occurred. Fourth, our long-term follow-up data (a minimum of 5 years and an average of 6.9 years) indicate that the effects of the intervention are durable.

Some notes of caution are also needed to clarify the generalizability of these results. It is important to acknowledge the fact that a larger cohort of patients would support the validity of these findings, while a randomized controlled trial would further strengthen internal validity. Moreover, it is also necessary to acknowledge that while CXL is a standardized procedure, MARK is not because it involves customized mini-incisions whose number, position, length, and 
Table 2. Preoperative and postoperative data for each patient, including average keratometry, central pachymetry, and visual acuity with glasses

\begin{tabular}{lllcccc}
\hline & $\begin{array}{c}\text { Kavg } \\
\text { (before) }\end{array}$ & $\begin{array}{c}\text { Kavg } \\
\text { (latest) }\end{array}$ & $\begin{array}{c}\text { Pachymetry } \\
\text { (before) }\end{array}$ & $\begin{array}{c}\text { Pachymetry } \\
\text { (latest) }\end{array}$ & $\begin{array}{c}\text { BSCVA } \\
\text { (before) }\end{array}$ & $\begin{array}{c}\text { BSCVA } \\
\text { (latest) }\end{array}$ \\
\hline Patient 1 & 43.5 & 39.25 & 415 & 421 & 2 & 7 \\
Patient 2 & 46 & 37.25 & 440 & 520 & 4 & 9 \\
Patient 3 & 53.5 & 50 & 309 & 311 & 4 & 6 \\
Patient 4 & 49.5 & 46 & 440 & 457 & 1 & 4 \\
Patient 5 & 46.5 & 55 & 460 & 464 & 2 & 3 \\
Patient 6 & 46 & 43.25 & 480 & 488 & 4 & 8 \\
Patient 7 & 42.25 & 39.75 & 449 & 454 & 8 & 9 \\
Patient 8 & 45.75 & 43.25 & 498 & 510 & 4 & 9 \\
Patient 9 & 46.5 & 47 & 564 & 548 & 2 & 4 \\
Patient 10 & 51 & 46.75 & 451 & 529 & 3 & 9 \\
Patient 11 & 46 & 44 & 512 & 543 & 4 & 8 \\
Patient 12 & 61.5 & 46 & 380 & 408 & 2 & 8 \\
Patient 13 & 52 & 44 & 393 & 430 & 4 & 9 \\
Patient 14 & 54.75 & 47 & 393 & 419 & 4 & 7 \\
Patient 15 & 47.5 & 45 & 458 & 466 & 5 & 8 \\
\hline
\end{tabular}

Kavg = mean keratometry; BSCVA = best spectacle-corrected visual acuity.

depth change with every patient. As a corollary, an ophthalmic surgeon with experience in incisional (both manual and laser) surgery is a prerequisite for this combined procedure.

The general availability of more accurate instruments for the diagnosis of $\mathrm{KC}$ is increasing, as well as awareness of this condition by the general public, which has resulted in diagnosis of a growing number of patients with KC. However, while medical research is progressing at an impressive pace and CXL alone is able to halt corneal ectasia, as showed by Snibson's review [22], the patient's visual acuity still needs to be improved, which justifies the 'CXL Plus' approach. Because every CXL-combined protocol has precise indications and limitations, as outlined by Mastropasqua [23], one can conclude that the more options the ophthalmic surgeon has, the better it is for their patients.

The rationale for combination of MARK and CXL is that the combined procedure can offer both corneal reinforcement and regularization, with the advantage of avoiding corneal thinning (PRK/FemtoLASIK) or intracorneal surgery (ICRS). Limitations include likely requiring glasses or contact lenses after surgery as well as very careful patient selection, as MARK is indicated only within the parameters that have been outlined previously. Rigorous inclusion criteria are essential to prevent possible unwanted complications deriving from inappropriate surgery, a number of which have been described in the literature and summarized by Lovisolo et al. [24].

With the positive outcomes and limitations of the Rome protocol outlined, it is possible to contextualize this research, as other CXL-combined procedures showed mixed results. Kanellopoulos and Asimellis [15] reported improved visual acuity and mean keratometry after performing PRK combined with CXL, but also decreased corneal thickness due to surface ablation. Similar findings were reported for the combination of LASIK and CXL by Kanellopoulos and Pamel [16]. El-Raggal [17], moreover, described improved vision and keratometry with ICRS combined with CXL, but did not discuss pachymetric changes. Toric phakic intraocular lenses, when implanted after CXL, also improved visual acuity and mean keratometry, while pachymetric values were relatively stable according to Fadlallah et al. [18]. Lastly, CKP followed by CXL was described as producing results no different from those with CXL alone [19]. The current literature on 
CXL-combined procedures, excluding CKP, therefore confirms significant gains in terms of visual acuity and mean keratometry, but also outlines varying outcomes relating to corneal thickness. MARK differs in this respect since it does not result in corneal thinning because there is no ablation and because post-keratotomy fibrotic cellular responses produce thicker collagen fibrils over the incisions along with corneal flattening, a process that has been widely discussed in the literature [25], without compromising the patient's vision due to the incisions' distance from the optical zone.

Lastly, some final considerations concerning MARK surgery are required for greater conceptual clarity. The differences between MARK mini-incisions and standard RK incisions are particularly significant when treating $\mathrm{KC}$, mainly due to the fact that MARK surgery: i) uses very short incisions (approximately $2 \mathrm{~mm}$ ) compared to fulllength RK incisions; ii) applies less deep incisions ( $80 \%$ of corneal thickness) than RK incisions; iii) requires few (3 to 5) customized mini-incisions that involve only a limited angular span of the cornea, as opposed to the full angular span involved with RK; and iv) does not preclude future surgical options such as penetrating keratoplasty, due to the fact MARK mini-incisions do not extend beyond a corneal diameter of $8 \mathrm{~mm}$ peripherally, which means that they would be included in the diameter of corneal tissue to be removed and substituted with the corneal graft. These are significant differences that are bound to affect long-term stability and safety.

We showed that the combined intervention of MARK and CXL (the Rome protocol) is effective at treating progressive $\mathrm{KC}$ and improving visual acuity based on analyses of long-term follow-up data (a minimum of 5 years and an average of 6.9 years). Mean keratometry and mean pachymetry improved in $87 \%$ and $93 \%$ of cases, respectively, while best corrected visual acuity increased in all cases. As mentioned previously, further studies with larger cohorts of patients are needed to confirm these results, and we also acknowledge the need for careful patient selection as well as experience with incisional surgery.

Surgical and parasurgical options for the treatment of $\mathrm{KC}$ keep increasing in number due to the need to comprehensively treat keratoconic patients, which is reassuring news for patients as well as for the medical and scientific community. In the wider context, it cannot be stressed enough that prevention is the best strategy for the treat- ment of this pathology, as an early diagnosis will likely result in early-stage CXL, which would probably prevent the need for further surgery. Moreover, 10 years' worth of follow-up data for CXL is available due to the study of Raiskup et al. [26], and this data further confirms the importance of treating progressive $\mathrm{KC}$ early and effectively. When this is not the case, however, the availability of multiple surgical options, such as the MARK + CXL procedure, as well as those outlined previously provides a wider treatment spectrum for the ophthalmic surgeon who can then perform the most appropriate one taking each patient's characteristics into consideration.

\section{Conflict of Interest}

No potential conflict of interest relevant to this article was reported.

\section{References}

1. Grzybowski A. Mauchart did not give the first description of keratoconus. Acta Ophthalmol 2014;92:e84-5.

2. Rabinowitz YS. Keratoconus. Surv Ophthalmol 1998;42:297319.

3. Oh BL, Kim MK, Wee WR. Comparison of clinical outcomes of same-size grafting between deep anterior lamellar keratoplasty and penetrating keratoplasty for keratoconus. Korean J Ophthalmol 2013;27:322-30.

4. Kelly TL, Williams KA, Coster DJ; Australian Corneal Graft Registry. Corneal transplantation for keratoconus: a registry study. Arch Ophthalmol 2011;129:691-7.

5. Wollensak G, Spoerl E, Seiler T. Riboflavin/ultraviolet-a-induced collagen crosslinking for the treatment of keratoconus. Am J Ophthalmol 2003;135:620-7.

6. Colin J, Cochener B, Savary G, Malet F. Correcting keratoconus with intracorneal rings. J Cataract Refract Surg 2000;26:1117-22.

7. Lombardi M, Abbondanza M. Asymmetric radial keratotomy for the correction of keratoconus. J Refract Surg 1997;13:302-7.

8. Abbondanza M. Mini Asymmetric Radial Keratotomy (Mini ARK) for the surgical correction of early-stage keratoconus, hyperopia, and mild myopia. Esperienze 1997;12:21-4. 
9. Krumeich JH, Kezirian GM. Circular keratotomy to reduce astigmatism and improve vision in stage I and II keratoconus. J Refract Surg 2009;25:357-65.

10. Alio JL, Claramonte PJ, Caliz A, Ramzy MI. Corneal modeling of keratoconus by conductive keratoplasty. J Cataract Refract Surg 2005;31:190-7.

11. Utine CA, Bayraktar S, Kaya V, et al. Radial keratotomy for the optical rehabilitation of mild to moderate keratoconus: more than 5 years' experience. Eur J Ophthalmol 2006;16:376-84.

12. Kocak I, Aydin A, Kaya F, et al. Efficacy of radial keratotomy in the optical rehabilitation of mild to moderate keratoconus cases. Int Eye Sci 2015;15:572-6.

13. Fujimoto K, Osawa H, Moriyama T, et al. Long-term stability of minimally invasive radial keratotomy for mild to moderate keratoconus. Asia Pac J Ophthalmol (Phila) 2017;6:407-11.

14. Abbondanza M, Abdolrahimzadeh B, Zuppardo M. Refractive changes following CXL. Cataract Refract Surg Today Eur 2009;4:33-8.

15. Kanellopoulos AJ, Asimellis G. Keratoconus management: long-term stability of topography-guided normalization combined with high-fluence CXL stabilization (the Athens Protocol). J Refract Surg 2014;30:88-93.

16. Kanellopoulos AJ, Pamel GJ. Review of current indications for combined very high fluence collagen cross-linking and laser in situ keratomileusis surgery. Indian J Ophthalmol 2013;61:430-2.

17. El-Raggal TM. Sequential versus concurrent KERARINGS insertion and corneal collagen cross-linking for keratoconus. Br J Ophthalmol 2011;95:37-41.
18. Fadlallah A, Dirani A, El Rami H, et al. Safety and visual outcome of Visian toric ICL implantation after corneal collagen cross-linking in keratoconus. J Refract Surg 2013;29:84-9.

19. Kymionis GD, Kontadakis GA, Naoumidi TL, et al. Conductive keratoplasty followed by collagen cross-linking with riboflavin-UV-A in patients with keratoconus. Cornea 2010;29:239-43.

20. Abbondanza M, Abbondanza G, De Felice V. Mini asymmetric radial keratotomy and corneal cross-linking for the treatment of a bilateral stage IV keratoconus in a 14-yearold child. Med Arch 2017;71:69-71.

21. Lindstrom RL. Minimally invasive radial keratotomy: mini-RK. J Cataract Refract Surg 1995;21:27-34.

22. Snibson GR. Collagen cross-linking: a new treatment paradigm in corneal disease: a review. Clin Exp Ophthalmol 2010;38:141-53.

23. Mastropasqua L. Collagen cross-linking: when and how? A review of the state of the art of the technique and new perspectives. Eye Vis (Lond) 2015;2:19.

24. Lovisolo CF, Mularoni A, Calossi A, et al. Complications of refractive keratotomy. In: Alio JL, Azar DT, editors. Management of complications in refractive surgery. Berlin: Springer; 2008. p. 197-224.

25. Wilson SL, El Haj AJ, Yang Y. Control of scar tissue formation in the cornea: strategies in clinical and corneal tissue engineering. J Funct Biomater 2012;3:642-87.

26. Raiskup F, Theuring A, Pillunat LE, Spoerl E. Corneal collagen crosslinking with riboflavin and ultraviolet-A light in progressive keratoconus: ten-year results. $J$ Cataract Refract Surg 2015;41:41-6. 